\section{Advanced Confocal Microscopy An Essential Technique for Microfluidics Development}

\author{
Terence Lundy \\ Hyphenated-Systems, Burlingame, CA \\ tlundy@hyphenated-systems.com
}

\section{Introduction}

Many believe that microfluidics has the potential to do for chemistry and biology what the integrated circuit has done for electronics - integrating tremendously complex chemical and biological processes into simple easy-to-use devices that will eventually pervade our lives. While microfluidics has made great progress in the last decade - addressing many of the fundamental questions related to manipulating nanoliter volumes of chemicals and solutions - it still faces some very basic challenges as it moves out of the laboratory and into use. Perhaps most basic is the need for fast, accurate characterization of the size and shape of the microfluidic devices themselves. Conventional imaging and measurement techniques have proven adequate for initial development, but are unable to provide the speed and accuracy needed to support the continued development of microfluidic technologies. This article will review existing characterization techniques to identify their strengths and deficiencies in comparison to the capabilities offered by advanced confocal microscopy (3Dmap, Hyphenated-Systems).

\section{Analytical Requirements for Microfluidic Development}

\section{Sub-micrometer resolution in three dimensions:}

Microfluidic devices are typically composed of an array of micrometer-scale features including channels, valves, pumps, mixers, and other essential components. The ability to measure and control the size and shape of these structures is a primary requirement, not only to ensure the functionality of existing devices, but also to provide a basic tool for discovering and developing novel designs.

\section{Sub-surface capability:}

In an approach similar to the electronic integrated circuit, the
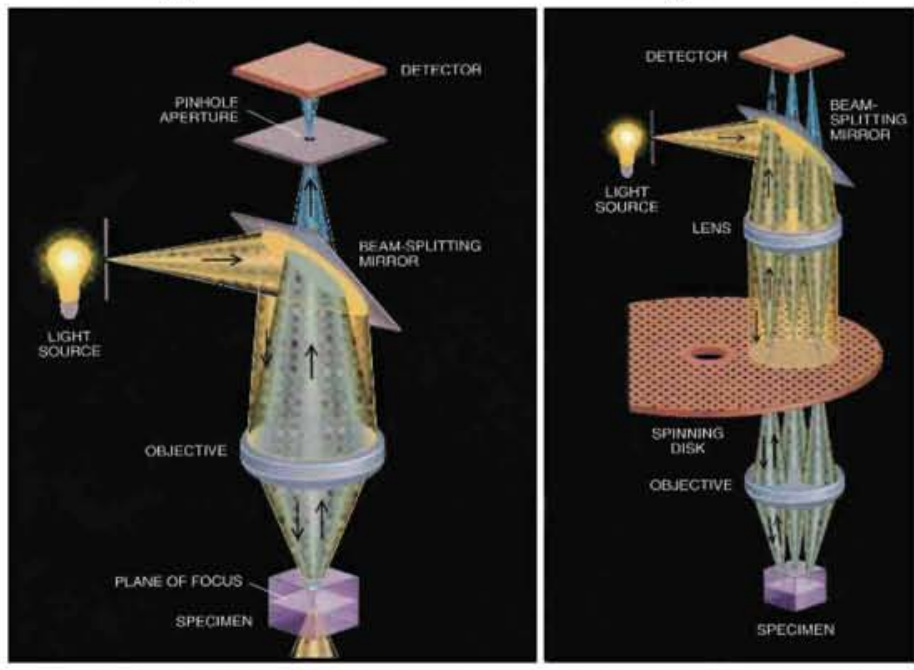

Figure 1. All confocal microscopes use an aperture to exclude light that originates above or below the object plane, giving them excellent depth resolution. However, the aperture also excludes information from all points in the object plane except the imaged point, thus requiring a scanning approach to image acquisition. Advanced confocal microscopy simultaneously acquires data from multiple apertures in a spinning disk to dramatically reduce image acquisition time. ACM can produce a detailed model with sub-micrometer resolution in all three dimensions in a few seconds.

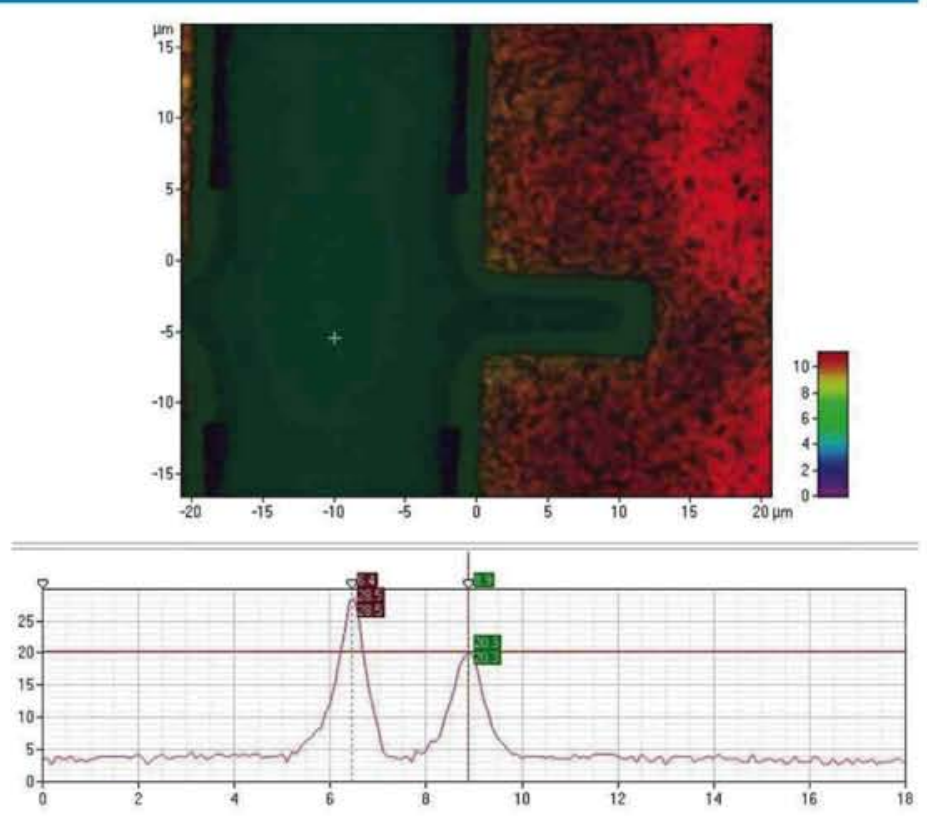

Figure 2. In this false-color, top-down image red areas are high, green and blue are lower. The graph shows the signal strength at the cursor position (white +) as a function of depth. The two peaks correspond to two surfaces at different depths.

various components of a microfluidic device are often arranged in one or more layers. This imposes an additional requirement for precise relative positioning of features within a layer and registration between layers. Moreover, characterization of a finished device requires the ability to look beneath the surface at buried structures.

Access:

In their efforts to fully understand the operation of their devices, researchers and engineers will often need to use other analytical tools, such as flow characterization or fluorescence techniques. The ideal platform should provide ample sample access and easy integration for these tools.

Speed:

Existing characterization techniques require many seconds to several minutes, or more, to provide results. Shortening that to a few seconds is clearly desirable, even in a research environment. However, as the microfludics industry moves from development to production, analytical speed in process control applications will be a critical determinant of productivity and profitability.

\section{Conventional Technologies Used by Developers}

Conventional optical microscopy has been, and will remain, an essential tool in microfluidics development. Though it can provide areal resolution at the sub-micrometer scale, it is limited in its ability to characterize structure in the third $(z)$ dimension. While it is certainly possible to focus on subsurface features within a thick specimen, it is not possible to exclude interfering information from above and below the plane of focus. Stereo microscopy techniques, based on combining images acquired from slightly different perspectives, are also incapable of excluding this interference.

Interferometry extracts three-dimensional information from patterns created when light reflected from the sample surface interferes with an unperturbed reference wave front. It can provide nanometer scale resolution in the $z$ direction. However, it suffers from a number of limitations in microfluidic applications. In a sense, it offers too much $z$ resolution. Even a mildly sloped surface can generate interference patterns that cannot be resolved and analyzed. Microfluidic structures often include sloped surfaces that exceed the method's capability. 


\section{RevolutionEDX}

Universal Spectral Engine ${ }^{T M}$

The leader in EDX and imaging systems innovation introduces the next revolution in microanalysis:

\section{RevolutionEDX}

The most significant advance in EDX since Spectrum Imaging.

EDX \& Imaging Systems
At the heart of RevolutionEDX ${ }^{\mathrm{TM}}$ is the Universal Spectral Engine: a new concept in Energy Dispersive X-Ray Microanalysis and Imaging.

See why we call this a revolution. Call us today to learn how integrating this remarkable new system into your workflow can save you time and add new capabilities and power to your electron microscope.

\section{Integrated by design}

All new electronics designed for high-speed transport of digital x-ray and electron event data

\section{The first Universal system}

A stand-alone network system that seamlessly interfaces to all $x$-ray detectors and SEMs/STEMs regardless of manufacturer

\section{Unique streaming technology}

Provides rapid 16-bit x-ray and electron image collection for real-time analysis

\section{Join Us}

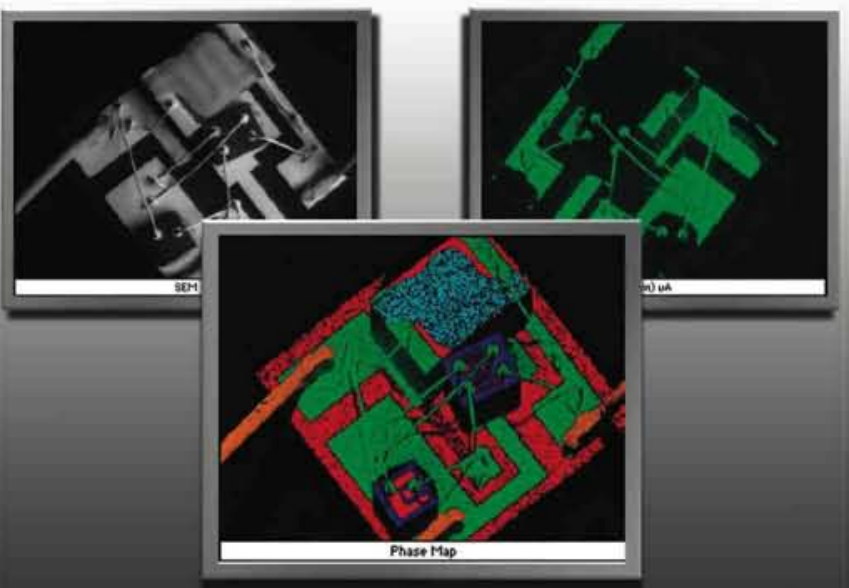

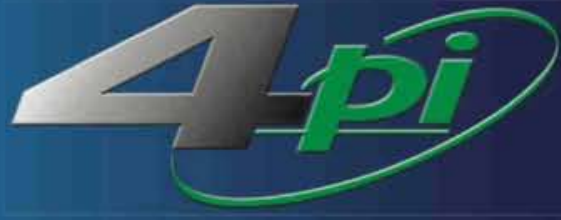

www. 4 ol.com

4 pi Analysis, Inc.

(919) 489-1757•info@4pi.com 

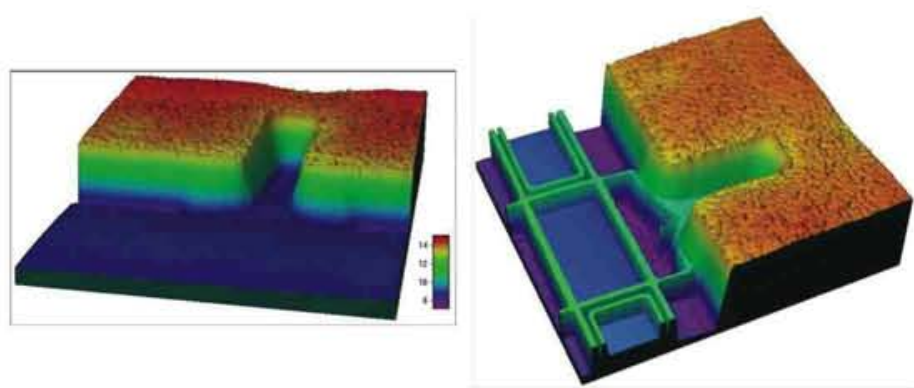

Figure 3. the same sample as figure 2 rendered in three dimensions. The blue surface on the left is a glass cover. On the right, the system has excluded the signal from the cover to reveal the structure below.

Generally, interferometry also requires the measured surface to be the first surface encountered by the incident light, thus precluding its application to subsurface structure. It has difficulty analyzing non-continuous surfaces and rough surfaces that scatter much of the incident light.

Atomic force microscopy and other scanning probe techniques have sufficient three-dimensional resolution for microfluidic applications, but are also "first surface" techniques with no access to subsurface structure and limited $z$-axis range. Furthermore, the sequential nature of their data acquisition makes them relatively slow.

Confocal microscopy addresses many of the limitations of the previously mentioned techniques. Like conventional optical microscopy, it uses visible light and can provide sub-micrometer areal resolution. Confocal microscopes include an additional aperture located in the
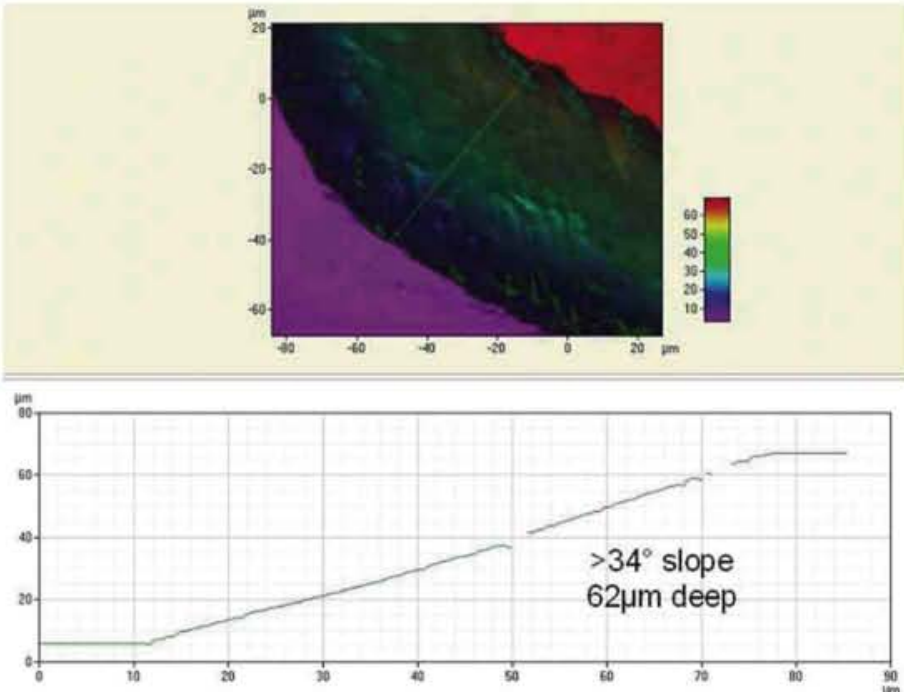

Figure 4. The edge profile of a channel etched in silicon. The slope and roughness of this surface would make it difficult to measure with interferometry.

imaging plane that admits only light from a very small volume in the sample. This arrangement gives confocal microscopy excellent resolution in the $z$ direction by excluding light from features above or below the object plane within the sample. Unfortunately, it also excludes light from all other points in the object plane, forcing a scanning approach to image acquisition. Though modern scanning techniques are relatively fast, the sequential nature of the data acquisition imposes a fundamental limit on the technique's speed.

Advanced Confocal Microscopy (ACM) circumvents the throughput restriction of scanned confocal techniques by providing parallel data collection from multiple points within the object plane (Fig 1). It is based on the Nipkow disk, originally invented as a means of producing scanned pictures for television. The disk contains multiple apertures arranged in a spiral pattern. It is located in the image plane of the microscope where it spins rapidly, such that each aperture traces a linear path through the image. A detector acquires data from each aperture and a computer assembles the data into an image. Spinning disk confocal microscopes have been available since confocal microscopy became popular decades ago; however, the similarities between early implementations and today's ACM stop there. The greatest advances have come not from the fundamental imaging technique, but from the explosive growth in data processing power. ACM can acquire a twodimensional slice through the sample in a small fraction of a second, and can assemble and render a high resolution three-dimensional model composed of hundreds of these slices in a few seconds.

Advanced measurement and analysis software also play an important role in ACM. For instance, imaging algorithms permits the operator to select which parts of the model to view, making it easy to view subsurface structure. Figure 2 shows a false-color areal image of the surface of a device. Red regions are high, green and blue are lower. Closer examination of the signal collected at the cursor position shows two peaks in signal strength occurring at different levels within the sample. (The graph represents intensity on the $y$-axis and depth on the $x$-axis.) In default mode, the rendering algorithm selects the strongest peak, in this case the glass cover layer at 6.4 micrometers, to render as the sample surface (Fig 3 left). Forcing the algorithm to ignore peaks above a certain level reveals the structure below the cover (Fig 3 right).

3Dmap brings the full power of ACM to bear on the problems confronting scientists and engineers in the fast developing world of microfluidics. It provides sub-micron resolution in all three dimensions. It can selectively image sub-surface structures in multi-layer devices, including rough, discontinuous and sloped surfaces. Its inverted optical design and open architecture provide easy access to the sample for other analytical techniques. Finally, it delivers critical results in seconds.

\section{Applications}

Microfluidics has not yet reached the stage where any single fabrication technology has established dominance. Many techniques are used in many different applications. They include milling, stamping, casting, laser ablation, and photolithography/etching, to name only a
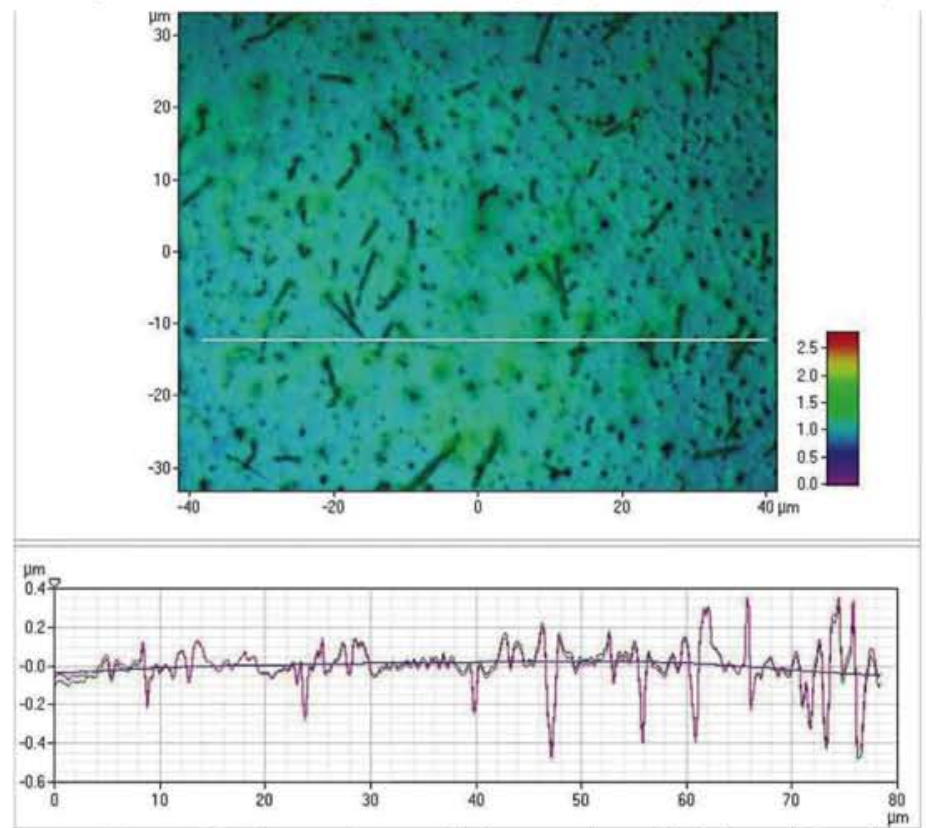

Figure 5. Surface roughness at the bottom of an etched channel. Rough surfaces often scatter too much light to be measured with interferometry. 
few. The materials used include glasses, silicon, metals, plastics, rubbers, biopolymers and more. Components may be as simple as a channel or as complex as a valve or a multi-chambered pump. Below we look at several features created using a variety of fabrication technologies.

\section{Flow Characterization}

The ultimate goal of microfluidics is the manipulation of very small volumes of fluid. In this size regime, and for most fluids of interest in microfluidic applications, flow is typically laminar. This can be advantageous, as for instance when it preserves the integrity of a bolus of fluid transported through the device. In other cases, mixing of fluids is desirable, and the device must be specifically designed to induce turbulence. In all cases, the micro-scale size, shape and surface characteristics of the device are critical to its performance, but the physical characterization of the device structure is only useful if we can determine its effects on the behavior of the fluids. This requires a means of visualizing the flow itself at the micrometer scale. A number of techniques are available that use dyes, bleaches and marking particles of various kinds.

Caged fluorescence uses a fluorescent dye that is initially in a "caged" non-fluorescent state. After the dye is introduced into the experimental flow, a pulse of ultraviolet light breaks the bonds that cage the fluorescent group. The initial distribution of the uncaged fluorescent marker is thus confined to only the region illuminated by the activating light. At some time after activation, the now fluorescent dye is excited by a brief pulse of laser light, and its distribution is captured by an imaging device. Figure 10 uses the caged fluorescence technique to demonstrate the difference in dispersion that occurs in flow driven by electroosmotic forces (left) and pressure (right). In electroosmosis, fluid molecules are ionized near the walls of the channel to form a bi-layer with one species of ion adsorbed to the channel wall and the oppositely charged species free to migrate in response to an imposed field. The movement of this sheath of ions is translated to the rest of the fluid through viscous forces and results in minimal dispersion. In contrast, pressure driven flow has zero velocity at the channel wall and maximum velocity in the center of the channel, causing significant dispersion over even short distances.

Bleached fluorescence is the converse of caged fluorescence. In bleached fluorescence techniques the fluorescent dye is converted to a non-fluorescent state by a photobleaching pulse from an argon laser. Figures 11 and 12 show the use of bleached fluorescence and numerical modeling, to analyze flow in a curved electrophoresis channel.

Electrophoresis is a widely used separation technique based on the different rates at which
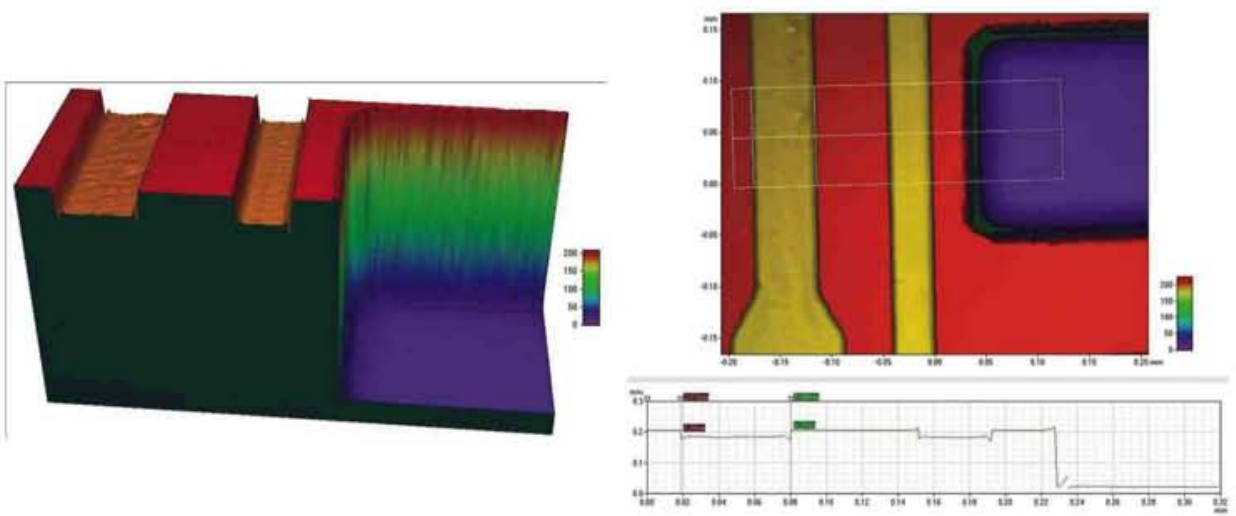

Figure 6. In casting processes a liquid material is poured over a mold, which is later removed. Notice in this image the small trenches at the junction of the channel floor and wall.
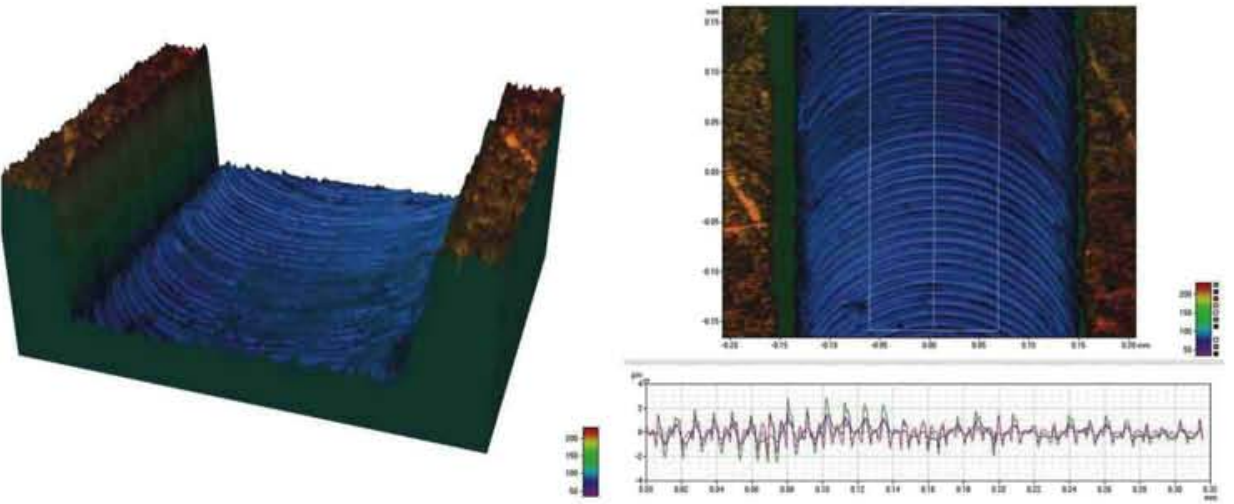

Figure 7. Micromachining uses traditional machining processes such as milling. In this case the milling marks are still apparent in the bottom of the channel. These will affect flow characteristics within the channel and may be desirable or undesirable.
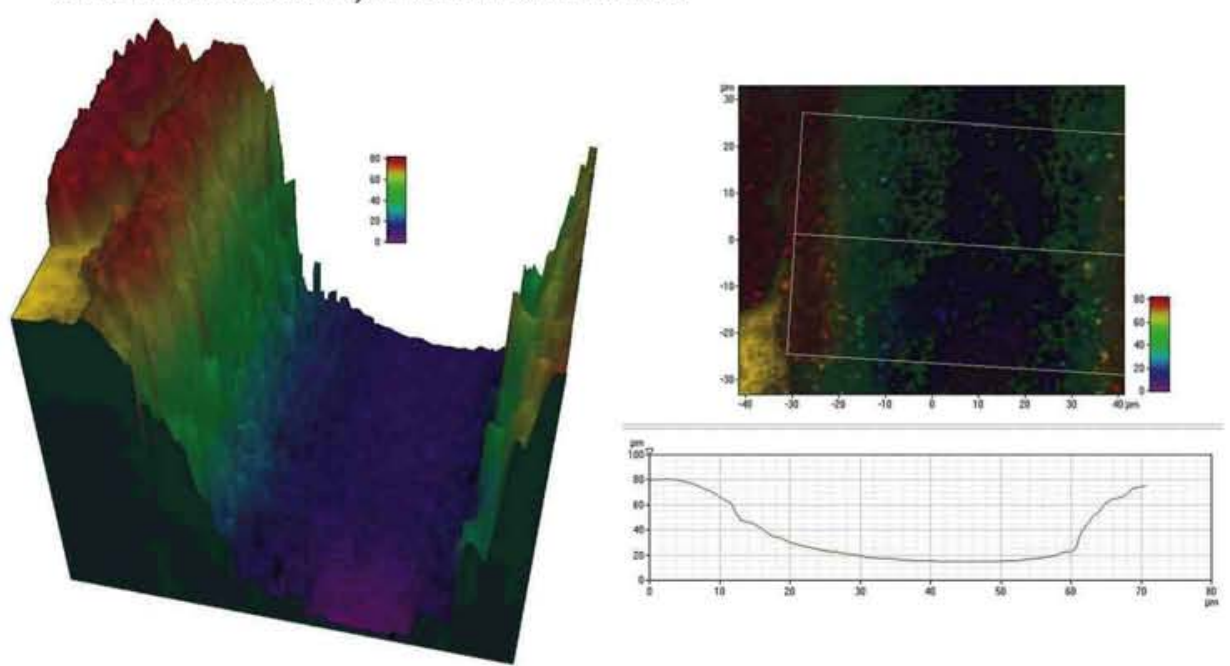

Figure 8. Laser ablation has the advantage of being able to create subsurface channels. The shape of the channel is clearly visible, and measurable, with ACM.
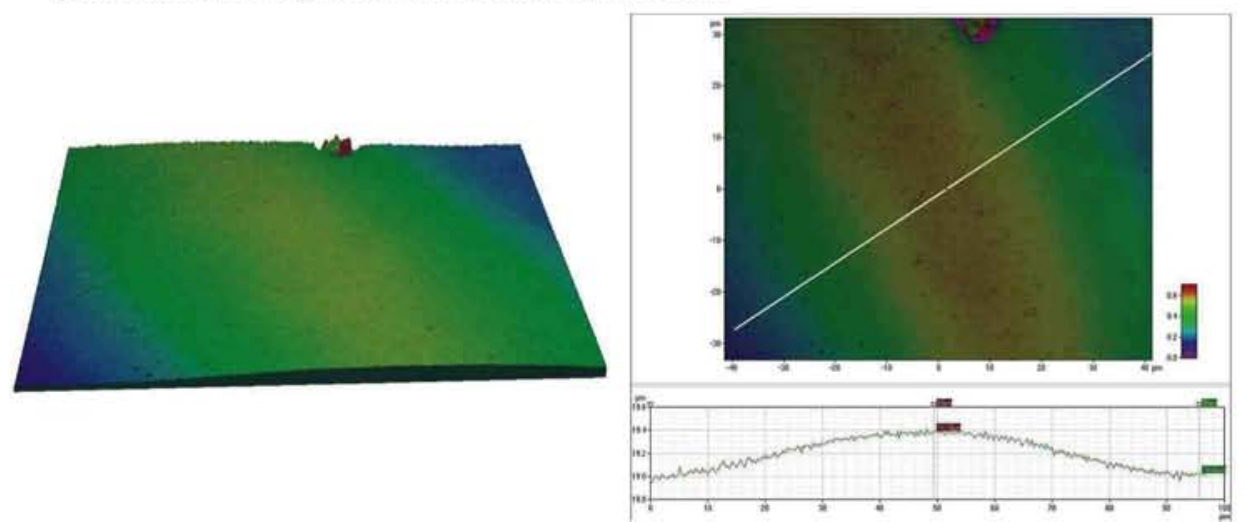

Figure 9. One approach to large scale integration uses arrays of pressure-activated micro-valves molded from flexible rubber compounds. This image shows the profile of a "bump" in the valve membrane that is critical to its operation. 

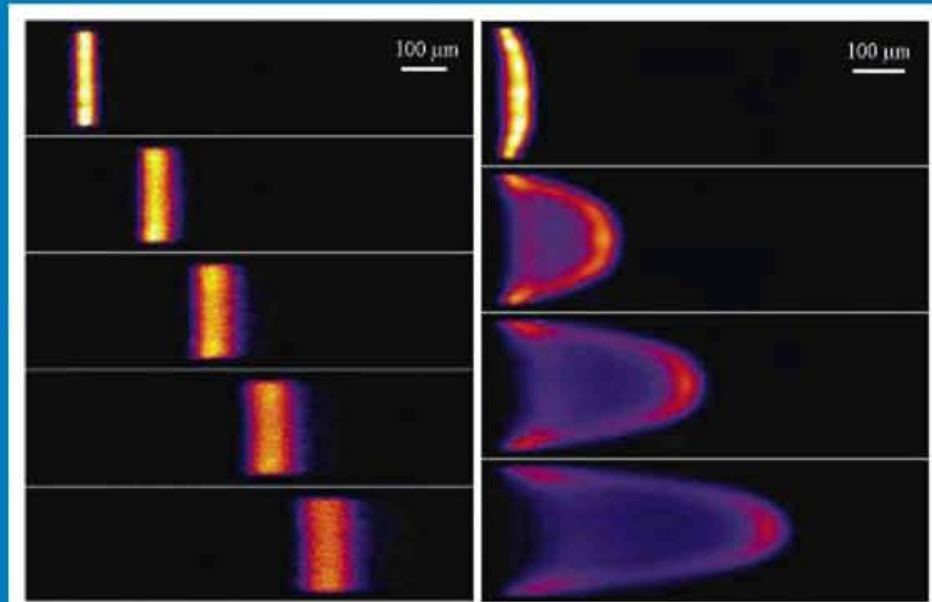

Figure 10. Caged fluorescence images demonstrate the difference in dispersion between electroosmotically-driven and pressure-driven flows ${ }^{1}$.

different sized molecules can travel through a molecular sieve material - usually a gel. Microfluidic implementations of capillary electrophoresis promise to reduce reagent volume and analysis time. However, achieving sufficient separation requires long channels - too long for most microfluidic "chips." The channel can be longer if it is folded, but bends in the channel introduce differences in path lengths through constant radius turns, which cause unwanted dispersion in the analyte. As shown in figures 11 and 12, modifying the inner radius of the turn reduces the unwanted dispersion. The figures compare a numerical simulation (top) with results of bleached fluorescence analysis.

Particle image velocimetry (PIV), and closely related particle tracking velocimetry (PTV), are sophisticated image analysis tech-
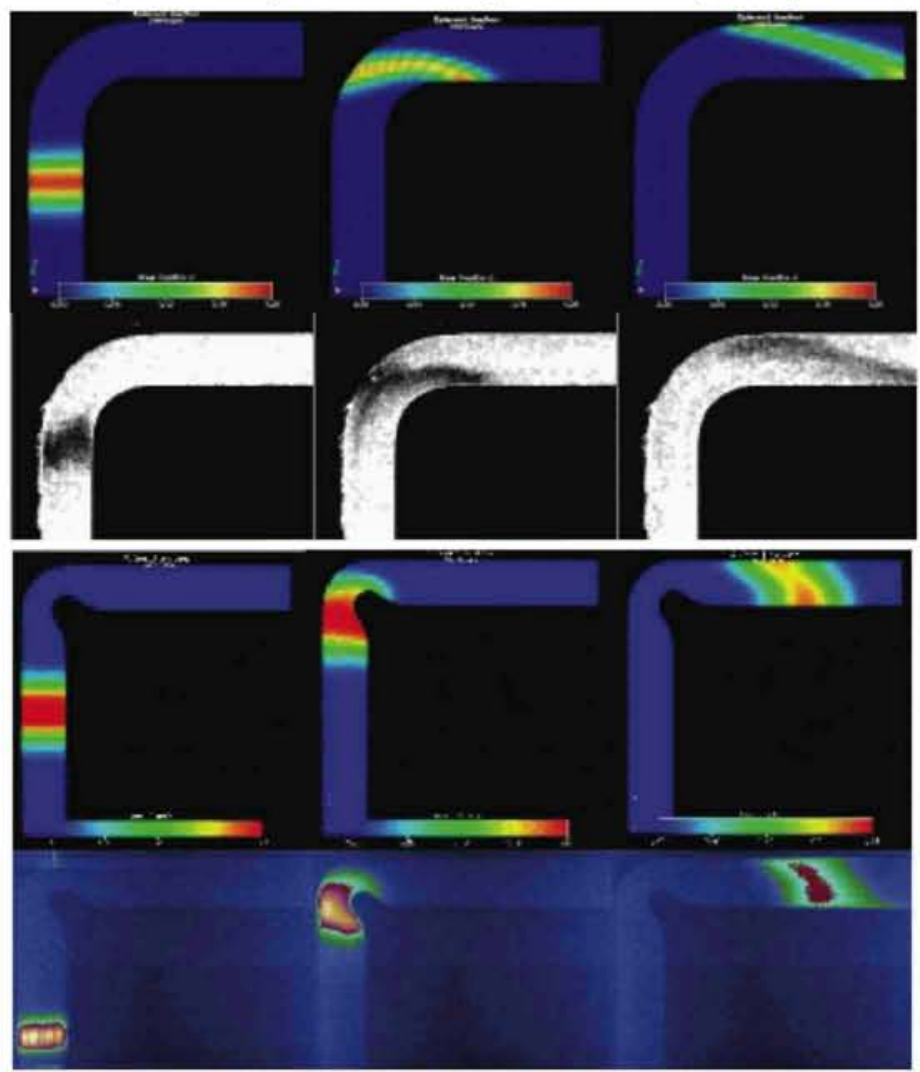

Figure 11. Top. Numerical simulation (top) and bleached fluorescence (bottom) analysis of dispersion caused by a constant radius turn in an electrophoresis channel 2,3 .

Figure 12. Bottom. Same as figure 11 showing reduced dispersion with modified turn geometry2,3. niques that characterize flows based on the movement of marker particles. PIV has been used in macro scale fluid dynamics for some time and has recently been adapted to the micrometer scale for microfluidic applications. Micro PIV cross-correlates sequential images of large numbers of marker particles to derive flow measurements. PTV actually tracks the movements of individual particles between images. It has the advantage of being able to separate the effects of random processes (such as Brownian motion) and particle interactions from the larger scale flow. Figure 13 shows the results of a PIV analysis of electrokinetic flow through a channel intersection. In this example the side channels were allowed to float electrically.

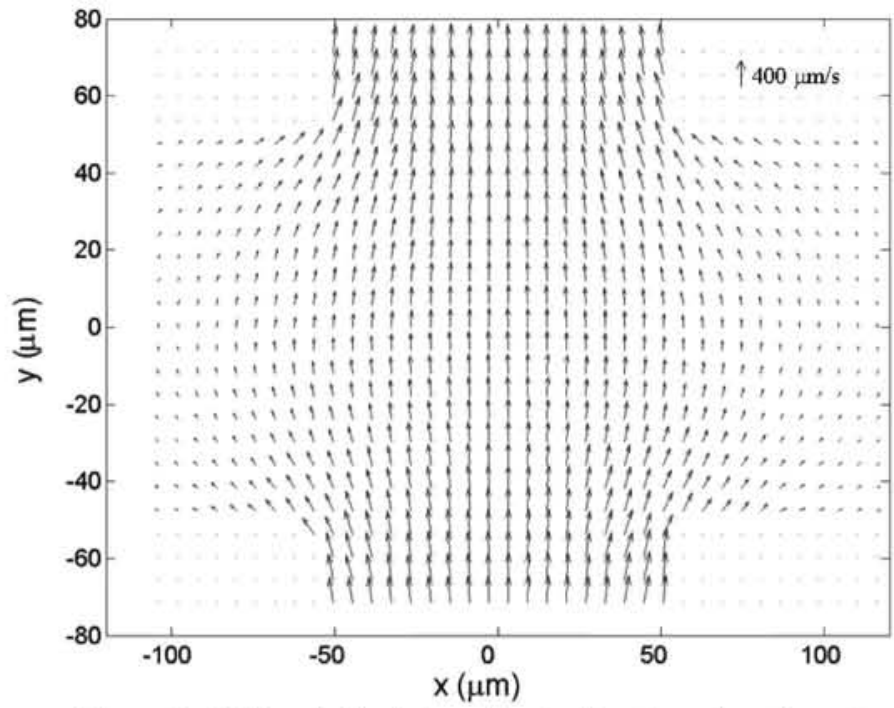

Figure 13. PIV analysis of electrokinetic flow through a channel intersection 4

\section{Conclusion}

Microfluidics has the potential to transform biology and chemistry in much the same way the integrated circuit revolutionized microelectronics. In addition to reducing sample volumes, reagent costs and analysis times, microfluidic devices can integrate complex biological and chemical processes in a simple lab-on-a-chip that may be reusable or even disposable. Fluids behave differently at the microscale and the structural details of microfluidic devices are critical to their performance. Researchers have been hampered by the lack of an analytical platform that can provide fast, accurate, high-resolution measurements, and that is flexible enough to integrate flow characterization tools and other analytical techniques essential to continuing development. Advanced confocal microscopy meets the analytical requirements, and its implementation in Hyphenated Systems' 3Dmap platform provides ample access for ancillary analytical techniques. This combination of fundamental measurement capability and open-ended analytical flexibility destines the 3Dmap platform to play a central role in continuing microfluidics development.

\section{References}

1. Molho, J. I. Electrokinetic Dispersion in Microfluidic Separation Systems. Ph.D.Thesis, Stanford University, 2001.

2. Molho, J.I., Herr, A.E., Mosier, B.P., Santiago, J.G., Kenny, T.W., Brennen, R.A., Gordon, G.B. and Mohammadi, B., "Optimization of Turn Geometries for OnChip Electrophoresis," Analytical Chemistry, Vol. 73, No. 6, 1350-1360, 2001.

3. Mohammadi, B., Santiago, J.G., "Simulation and Design of Extraction and Separation Fluidic Devices," Mathematical Modelling and Numerical Analysis. Vol. 34 , No. 3, 513-523, 2001.)

4. Devasenathipathy, S.; Santiago, J. G.; Takehara, K. Analytical Chemistry 2002, 74,3704 . 


\section{Model 950}

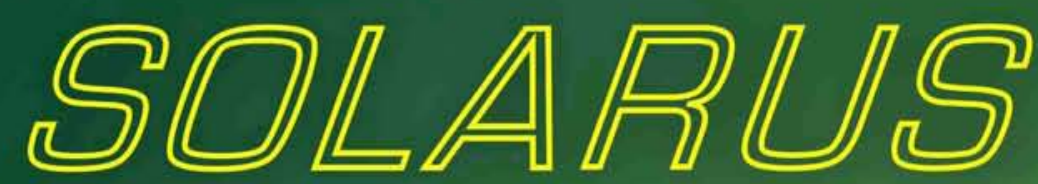

\section{Advanced Plasma System}

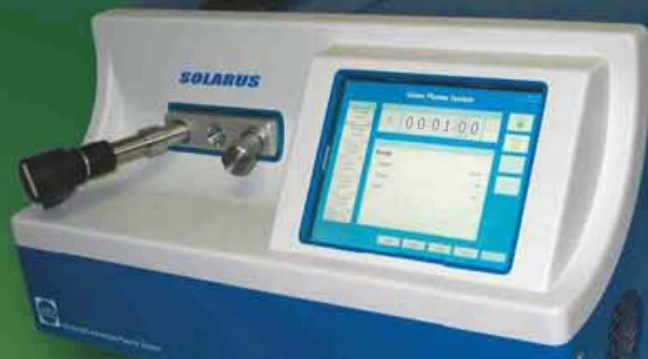

FAST EFFECTIVE DAMAGE-FREE GLEANING

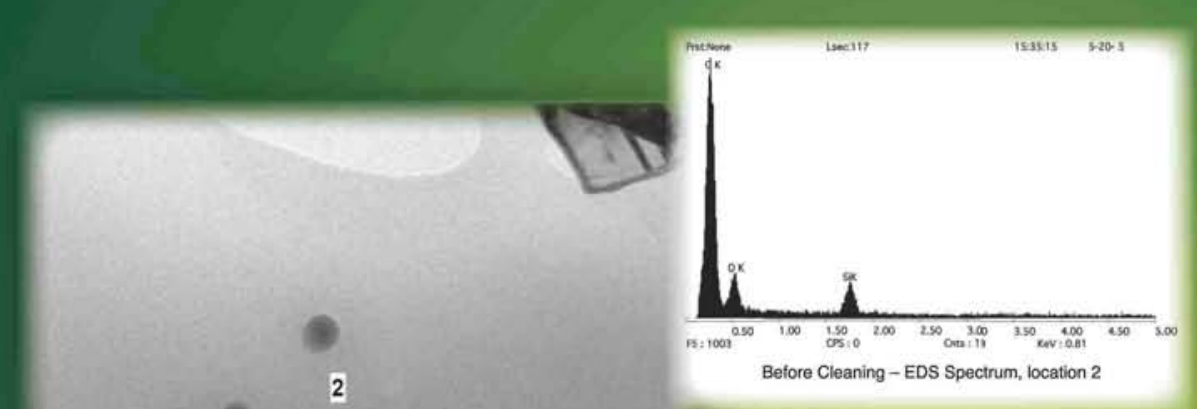

1

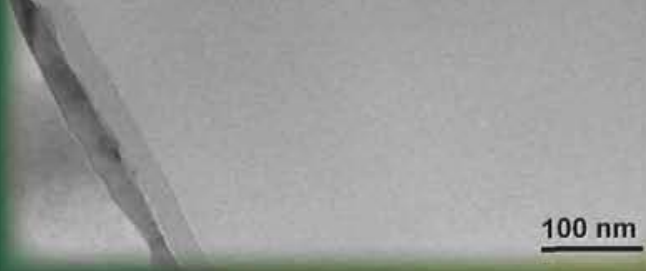

Before Cleaning - Wedge polished silicon sample +10 min PIPS ion milling. Contamination grown during spectrum collection times of $74 \mathrm{sec}$

\section{Cleans TEM/SEM SAMples}

Unigue $\mathrm{H}_{2} \mathrm{O}_{2}$ Cleaning Recipe [Patent Pending]

Safe Cleaning of Holey Carbon Films Ab/O $\mathrm{O}_{2}$ Cleaning Recipe Shortest Cleaning Cycle Times

85W RF POWER SUPPLY AND AUtomatch RF SYStem for Consistent Results

Touch Screen Technology After Cleaning - EDS Spectrum
181 sec collection time, location 3

$3^{4}$

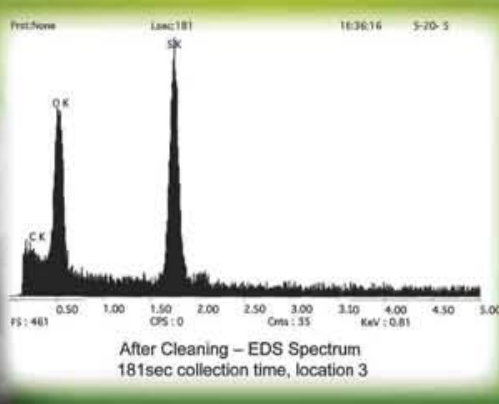

ONE-TOUCH RECIPE SELECTION Front and Top LoAding Chamber

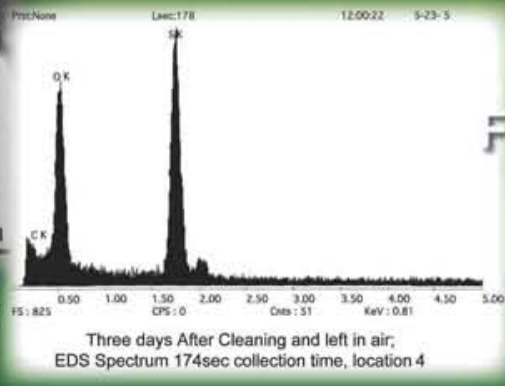

